\title{
Use Study on a Home Video Editing System
}

\author{
Hans Weda \\ Philips Research Europe \\ High Tech Campus 34 \\ 5656 AE Eindhoven, The Netherlands \\ +3140 2747939 \\ hans.weda@philips.com
}

\author{
Marco Campanella \\ Philips Research Europe \\ High Tech Campus 34 \\ 5656 AE Eindhoven, The Netherlands \\ +3140 2747899 \\ marco.campanella@philips.com
}

\begin{abstract}
To help consumers dealing with their growing amount of home video, we have developed the Edit While Watching (EWW) system. It is designed to automatically create an edited version of a home video and then allow the users to modify and refine it in an easy, intuitive and lean-back way. To measure the ease of use, ease of learning, and effectiveness of the EWW system, we have performed a use test by means of giving participants tasks to do and interviewing them. The use test was focused on four main aspects: functionality, usability, pleasantness, and user satisfaction. The test was performed with eight participants, and was located in the Philips HomeLab, which resembles a home environment as much as possible. The results show that the system provides rather limited control of the editing functions, and the overview of the video material is unsatisfactory. However, the participants judged the system as an easy to learn and easy to use video editing tool. They expressed their pleasure in working with it.
\end{abstract}

\section{Categories and Subject Descriptors}

H.5.2 [Information Interfaces and Presentation]: User Interfaces - User-centered design, evaluation/methodology.

\section{General Terms}

Design, Experimentation, Human Factors, Verification.

\section{Keywords}

Intelligent user interfaces, user experience evaluation, home video editing.

\section{INTRODUCTION}

In recent years, more and more people capture their experiences in home videos. While the market of digital camcorders is stable, the number of gadgets with an embedded camcorder is rapidly increasing: nowadays almost every mobile phone has a camcorder that can shoot medium to good quality videos.

Although the technology for capturing home videos is becoming cheaper and more efficient, the tools for enriching and editing the users' videos are still basic. Because video media consists of both a visual and an audio stream, and each stream contains many frames every second, video media is intrinsically complex and difficult to process, manipulate, and

(C) Hans Weda, Marco Campanella, 2007 Published by the British Computer Society Volume 2 Proceedings of the 21st BCS HCI Group Conference

HCI 2007, 3-7 September 2007, Lancaster University, UK Devina Ramduny-Ellis \& Dorothy Rachovides (Editors) share with others. This makes video editing a difficult and timeconsuming task [5].

To resolve this issue, the Edit While Watching (EWW) system has been developed [3]. It has been designed to automatically create an edited version of a home video and then allow the users to modify and refine it in an easy to use and lean-back way. After uploading the raw video material, the video is indexed, segmented, shortened, and combined with proper music and editing effects. This is based on content analysis [4][9]. The result is an automatically generated edited version of the home video that is shown to the user. While watching it, users can indicate by using a remote control whether they like certain content, so that the system will adapt the edited version to contain more content that is similar or related to the displayed content. EWW does not require a complex user interface: a TV and a few keys of a remote control are sufficient.

To assess the degree of ease of use and ease of learning of the design we have performed a use test on the EWW system. The test consisted in giving the participants practical tasks to do (i.e. editing a home video) and interviewing them. The focus was on four main aspects: functionality, usability, pleasantness of the system, and the user satisfaction while working with the system.

This article is structured as follows. The next section describes the EWW system in more detail. Section 3 is devoted to the definition of the objective of the test. The implementation of the use test is discussed in Section 4. The results and conclusions of the use test are dealt with in Sections 5 and 6 respectively.

\section{EDIT WHILE WATCHING SYSTEM}

Existing tools for video editing can be mapped onto two main categories: fully manual, frame-accurate video editing software programs ${ }^{1}$ and fully automatic summarization tools. A host of fully manual PC-based home video editing programs such as Adobe Premiere [2], and Pinnacle Studio [8] are already available on the market. These tools are based on low-level editing operations, but, to achieve nice results, users need to acquire quite some technical skills, have knowledge of the technical structure of a video document (frames, shots, transitions, filters) and invest a lot of time and effort. These solutions are ideal for people who want to have full control of their video to obtain a perfect result, but discourage most of the common users.

On the other side of the spectrum, some fully automatic solutions are present. In these software packages, the technical

\footnotetext{
${ }^{1}$ There are also video editing tools on devices such as hard disk recorders, for example [11]. These tools usually have limited automation, and therefore require considerable effort to use.
} 
details are hidden while users can still convey their own style and wishes through a simplified editing process. An example of these is Muvee autoProducer [7] or the function "smart movie" of Pinnacle Studio. After investigation and experiencing these systems, we found out that they do not provide enough control on the editing choices and processes. Users can only choose which raw material to use, specify the duration of the final result, and select a desired "style" (e.g. music video, romantic, slow-paced), then the tool produces the final video of the defined length. This final video consists of automatically selected parts of the raw material. Depending on the original duration of the selected raw material and the desired output length, the raw material is either partially used, or repeatedly shown. Users have no idea what the consequences of their choices are and cannot influence the "hidden" editing operations.

The EWW system we have developed overcomes the disadvantages of the existent solutions, by giving back control to the user via an easy to use interaction paradigm while hiding unnecessary technical details [3]. This is achieved by applying content analysis and film grammar rules to translate high-level users' choices into low-level editing operations. The EWW concept is targeted at the niche that is not yet occupied: easy home video editing in the living room.

The EWW system takes the raw video data as input. It extracts low-level features from the material such as camera motion, contrast, and luminosity. Based these low-level features the raw video data is segmented into short segments of typically 1-10 seconds. Each segment gets a numerical suitability score based on the mentioned low-level features. The segments with the highest scores are selected for the initial edited version, and shown to the user. The selected segments that are contiguous in time form a shot. Each editing operation affects one shot at a time.

Even the best automatic algorithm will never fully meet the desires of the user, since the relevance of a certain video scene is subjective and depends on the user's mood and taste, the experience recorded and the recipient of the video. Therefore, we aim at reaching the best edited version of the video using a synergy of human and machine abilities, providing the user with efficient interaction functionalities to refine the video.

The EWW system is not PC-based: the user interacts with it using a remote control and a TV-set. While watching, the user can press the pause button and select one of the following options (see Figure 1):

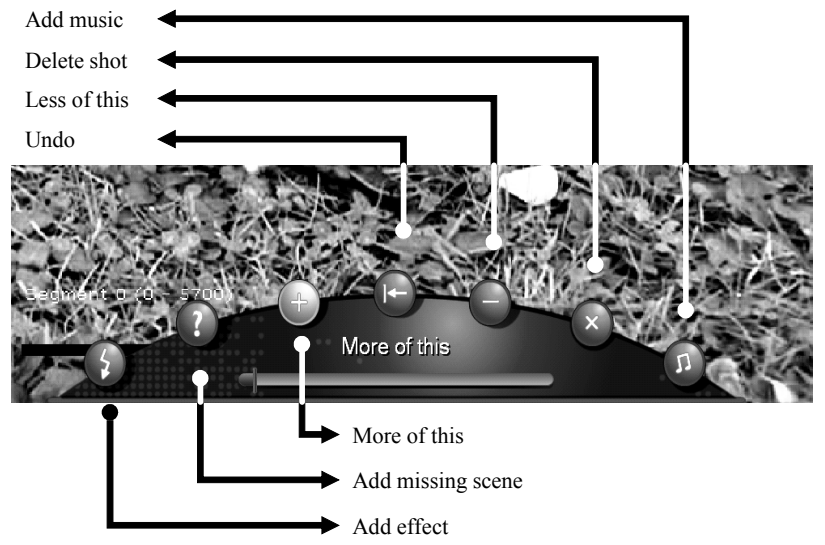

Figure 1. A screenshot of the user interface that appears at the bottom of the screen when the user presses 'pause' on the remote control to start editing.
- Add music to the current shot: shows a menu with different pieces of music to include in the current shot. Presently the option is limited to only five different music pieces. The idea is that, in a next version of EWW, the user can also upload his own music.

- Delete the current shot: deletes all segments of the current shot from the edited version.

- Less of this: deletes one segment (with lowest suitability score) of the current shot.

- More of this: adds one segment (with the highest suitability score) to the current shot.

- Add missing scene: shows a menu with different scenes not yet included in the edited version.

- Add effect: shows a menu with different video effects which can be applied to the current shot. Presently the effects are limited to about a dozen colour effects.

With the more and less of this functions the user can add or remove video content about a specific part by just pressing a button. The system will automatically add or remove video segments concerning that event from the edited version of the video.

By pressing the add missing scene button, the user is prompted with the thumbnail panel shown in Figure 2. Each thumbnail represents a scene of the raw material that is not included in the edited version. The user can browse these thumbnails and select the desired one.

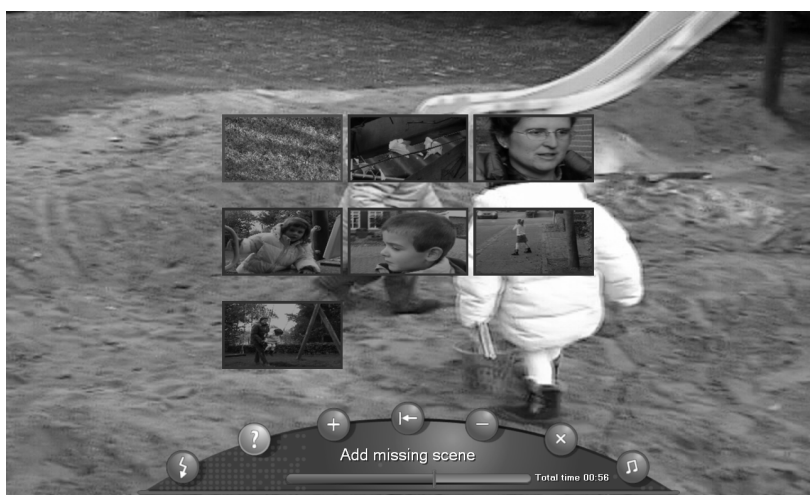

Figure 2. Screenshot of the "Add missing scene" menu

The button Undo, shown at the centre of the menu, is not yet implemented. Besides these listed editing options, we have implemented some navigation options in the version used for the use test. The options consisted of 'play' (at normal speed) and 'pause'. Furthermore the user can jump one shot ahead or back by using the arrow keys on the remote control.

Details on the technical implementation can be found in [3].

\section{OBJECTIVE OF THE TEST}

The objective of the use test described here is to assess whether EWW really enables the easy home video editing mentioned above. With respect to the functionality, we address the question if the system has sufficient options and functionalities for (basic) home video editing. Concerning the usability of the system, we investigate if the user understands the options and knows how to use them. Furthermore we investigate if the feedback of the system is clear to the user. 
We also study the satisfaction of the user when working with the EWW system. Do the users like to work with the system, and are they satisfied with the functionalities and the results?

\section{TEST DESIGN}

To answer the mentioned research questions we have divided the test in three parts: first we investigate the user behaviour and experience with respect to home video capturing, editing and sharing. In the second part of the test, the user is invited to try and use the system. We did not provide a full explanation of the functions. Therefore, initially the user is left as much time as needed for learning and getting acquainted with the system and its functions. Successively, the user is given a list of specific editing tasks to perform, like adding more video content about a certain event or delete the scene with an unwanted event. When the tasks are completed, the user is left as much time as wanted to freely edit this material according to his or her creativity. In the third part of the test, the user can give feedback on the system by means of a questionnaire and Likert scales [6], and an interview with the test leaders.

Since the EWW system is designed to be used in a home setting, we have selected the Philips HomeLab as test environment [1]. The HomeLab contains a living room with video cameras that recorded the test for later reference. A remote control and a TV set have been used for the interaction. A picture of the setup can be seen in Figure 3. All the operations done on the EWW program are logged for future reference. The test has a maximum duration of $60-90 \mathrm{~min}$.

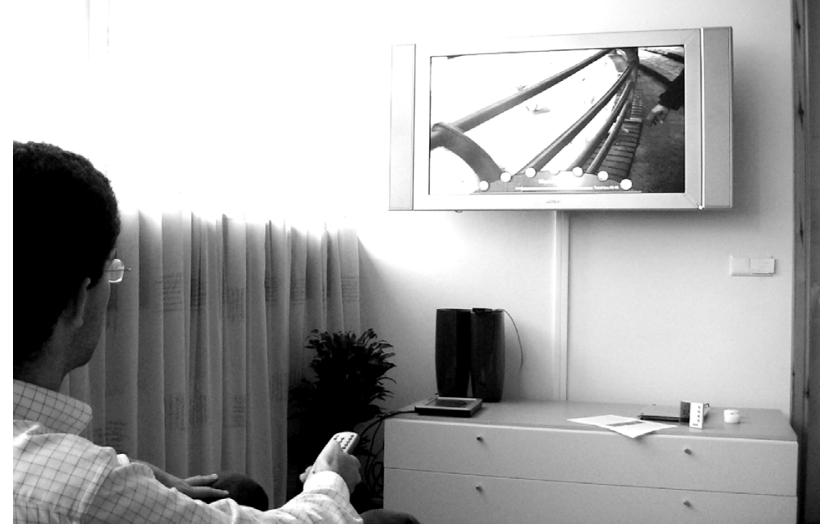

Figure 3. A picture of the test setup.

We have decided to use the same video material, which consisted of a home video of a Zoo visit, for all participants. The clear advantage is that all participants used the same material, and we can assign to the users specific editing tasks to perform, like adding more of the panthers and less of the bears in the final edited version. This allows us to evaluate the system with respect to particular tasks, which correspond to welldefined user requirements. The underlying assumption is that we know and understand how the system will typically be used. A disadvantage is that the test may seem artificial to the participant: he does not feel involved with the provided material. As a consequence, we cannot precisely investigate what the user needs are in editing his own material. However, from the test we found that the users considered the task scenario realistic. Therefore we feel confident about using the same video material for all users.

A usability test with four or five participants will expose 80 percent of the problems of the system, and this 80 percent represents most of the major problems [10]. To be certain to find the most apparent problems, and to gain some confidence, we invited eight participants.

\section{RESULTS}

\subsection{User Profile}

Eight students and employees from Philips Research in Eindhoven, with some experience in home video capturing, participated in the test: three females, and five males. None of the participants were working on related topics. Their age varied between 22 and 29, most of them capture video four or five times a year (range 2-100 times a year). When capturing video, six of the eight participants (6/8) use a digital still camera, or occasionally a camcorder (5/8). All of the participants own a digital still camera with video capturing functionality, half of them own a cell phone with video capturing functionality, and only two own a camcorder.

The captured video is rarely or never edited (7/8). Adobe premiere is the most used program for editing $(2 / 8)$. When editing is done, it takes a lot of time, 1 hour -1 day for 1 minute of edited video. Some participants have no video editing experience at all $(3 / 8)$. The video is mostly watched directly after capturing with friends, and only rarely shared or watched later. The explanation could be that usually unedited video is unattractive for these purposes, and video editing is too long and difficult to do.

\subsection{Questionnaire}

After the practical editing tasks, the user is asked to fill in a questionnaire on the functionality, usability, pleasantness, and user satisfaction with respect to the usage of the system. The questionnaire was printed on paper and involved twelve statements. The answers to these statements were given on Likert scales, ranging from 'false' to 'true'.

The results show that most participants agreed that the EWW system enables fast and easy editing. Furthermore, they mostly liked working with the system, and judged the task scenario as realistic. However, the final edited version did not convincingly satisfy the participants. The probable reasons are that the current editing functions are too limited and do not provide enough control. Additionally, the results show that there was a demand for more editing functions to further enrich the edited version. Furthermore, some users remarked that they would like to personalize the video by adding text, and inserting special transitions between the shots. Most of the participants do not outspokenly want to have the system, or to use it for their own home video.

\subsection{Remarks of the participants}

We have asked the participants to think aloud during the test. During their experience with the system and the final interview, users also expressed a number of free comments and remarks on the advantages and the deficiencies of EWW. Their comments and actions have been written down, and recorded on video. We have grouped and analysed the records. Based on this analysis, the following main results have been extracted.

Many users found the "more" and "less of this" functions too inadequate for their needs and difficult to understand. In general users were not satisfied by the fact that the system was automatically choosing which video segments to add or to discard for each more or less of this operation. Five over eight users would like to be able to decide whether to shorten or grow a scene from the beginning or from the end, three over 
eight would like to select how many seconds of video to add or to discard.

The system proved to have easy and effective navigation functionalities, all users but one found out how to navigate among the scenes by using the arrows. However, 4 over 8 users observed that fast-forward and rewind functionalities are missing and would be needed. Three over eight users expressed the lack of an overview of the whole raw footage, for example a key-frames panel or a timeline. They were, in fact, unfamiliar with the raw material and had difficulties to get acquainted to that using only the "add missing scene" functionality.

The "add missing scene" functionality was too limited to easily find a missing scene. One of the practical tasks consisted of adding a specific scene to the edited version that was not yet there. Five of the eight users expressed their difficulty in finding this specific scene.

As general remarks, 6 users were willing to use EWW or an improved version of it to edit their own video material. Everybody agreed that EWW is easy to learn and use, however 4 users clearly said that the system is too rigid and that they would like to have more control on the parameters of the editing operations and more editing functionalities.

\subsection{Log-files analysis}

During each test, we kept trace of the operations that users performed on the system by saving them into log files. From these files, some characteristics of the usage of the system can be extracted. The users employed on average 9 minutes (range $6-16$ minutes) to get acquainted with the system and to feel ready to start the tasks. These tasks were completed on average in 14 minutes (range 10-18 minutes). The average time in which the users enjoyed editing the video according to their own creativity was on average 8 minutes (range $3-15$ minutes).

Participants spent on average a large time using the system, dealing with it in a very personal way. The average time dedicated to creatively edit the video is about as long as the time which is spent on getting acquainted with the system. This fact, combined with the results from the questionnaire, seems to suggest that the users liked the system and enjoyed trying it out and spending time on it.

Users executed the tasks quite effectively, and all of them managed to complete them correctly. Only one or two users needed more time for completing some of the tasks. As expected, the two most used editing operations are "more" and "less of this". These were used more than twice as much as any other operation.

\section{CONCLUSIONS}

We have performed a use study on the EWW system. This system was designed as an easy to learn and use video editing system using just a remote control and a TV-screen. The test was performed with eight participants, who all had some experience in capturing video. The participants regarded the use and task scenario as realistic. They judged the system as an easy and fast video editing tool. It took the users a short time to try and learn all the functionalities implemented in the system. With regard to this aspect, the requirements of easiness and simplicity of EWW are met.

However, most users did not feel to be in control of the system. The lengthening and shortening of scenes seemed random to them, and they could not control the start and end of the added music precise enough. Furthermore they missed an overview of the material. It was regarded as difficult to easily see which parts were included and where, and what was left out. This could also be caused by the fact that the material was not their own, and consequently they were not familiar to all the content. Still the system was judged as reasonably 'fun' to work with. The users liked the system and liked to try it out and spend time on it.

Since the participants have a technical background and are highly educated, there is the danger of bias in the results. Participants with such a profile are typically fast learners and easy adopters. Furthermore they may tend to accept less surprises and unpredictability in the system, and would like to have more overview and control. On the other hand, all the non-technical people we have initially approached for the test were excluded due to lack of video capturing experience. Therefore the participants may be the interesting group to target with such systems. Therefore we think that the main deficiencies of the system found during the use test, are valid and relevant.

In the design of video editing systems, there seems to be a trade off between the ease of use and the amount of user control. Currently, systems allowing the user to edit video on frame level are difficult to learn and use. On the other hand, fully automatic systems are easy to use, but users cannot control all the details in the editing. With respect to these points, an optimal video editing system should try to optimize the balance between ease of use and user control. Thus, in an improved system, the ease of use, and ease of learning of EWW should be carefully preserved, while the user control and overview should be improved. Future work will therefore be concentrated on providing an easy to understand and use overview based on intuitive timelines, and on controllable trimming, deleting and adding of video.

\section{REFERENCES}

[1] Aarts, E., and Diederiks, E. (Eds.), Ambient Lifestyle, from concept to experience, Kluwer Academic Publishers, 2007.

[2] Adobe Premiere, http://www.adobe.com/products/premiere/

[3] Campanella, M., Weda, J., and Barbieri, M. Edit while watching: home video editing made easy. In proc. SPIE vol. 6506, 65060L, 2007.

[4] Furht, B., and Marques, O. (Eds.), Handbook of video databases: design and applications, CRC Press, 2004.

[5] Lienhart, R., Abstracting Home Video Automatically, Proc. ACM Intern. Multim. Conf., part 2, pp. 37-40, 1999.

[6] Likert, R. A Technique for the Measurement of Attitudes. Archives of Psychology 140, 55 (1932).

[7] Muvee autoProducer, http://www.muvee.com/

[8] Pinnacle Studio, http://www.pinnaclesys.com/

[9] Rosenfeld, A., Doermann, D., and DeMenthon, D., (Eds.), Video Mining, The International series in Video Computing, Kluwer Academic Publishers, 2003.

[10] Rubin, J. Handbook of usability testing. John Wiley \& Sons, 1994.

[11] Yasutaka, N., and Kyoko, T., Patent application, EP 1276 110, Pioneer Corp., 2003, http://gauss.ffii.org/PatentView/EP1276110 